\title{
MODELLING AND IDENTIFICATION OF ROBOTS WITH BOTH JOINT AND DRIVE FLEXIBILITIES
}

\author{
Toon Hardeman, Ronald Aarts, Ben Jonker \\ University of Twente \\ Faculty of Engineering Technology, The Netherlands \\ t.hardeman@ctw.utwente.nl
}

\begin{abstract}
Modelling and identification of flexible-joint robots is required for dynamic simulation and model based control of industrial robots. A nonlinear finite element based method is used to derive the dynamic equations of motion in a form suitable for both simulation and identification. The latter requires that the equations of motion are linear in the dynamic parameters. For accurate simulations of the robot tip motion, the model should describe the relevant dynamic properties such as joint friction and flexibilities. Both the drive and the joint flexibilities are included in the model. Joint friction is described by means of a static friction model, including Coulomb and viscous friction components. The dynamic parameters describing mass, inertia, stiffness, damping and friction properties are obtained from a least squares solution of an over determined linear system assembled from closed loop identification experiments. In the identification experiment, the robot moves along a prescribed trajectory while all joint angles, flexible deformations and driving torques are recorded. To excite joint vibrations during the identification feed forward torques at frequencies above the bandwidth of the control system are superposed on the joint torques. The applicability of the method is demonstrated in a numerical study of a four-link industrial robot.
\end{abstract}

Keywords: Parameter identification, flexible / elastic-joint robots, nonlinear finite element method

\section{Introduction}

Dynamic simulation and model based control of industrial robots require a correct description of the equations of motion and an accurate knowledge of the dynamic parameters. Determination of the dynamic parameters by using CAD data may not yield a complete representation as it is difficult to include dynamic parameters such as joint friction, masses introduced by additional equipment and stiffness parameters. Experimental parameter identification us- 
ing the assembled robot may be the only practical method to determine accurate values of the dynamic parameters.

The dynamic parameters can be identified by using a linear least squares method, provided that all degrees of freedom are measured during the identification experiment. Industrial robots are usually only equipped with motor encoders that measure joint angles. This implies that additional sensors are required to measure flexible joint degrees of freedom, e.g. by means of acceleration sensors (Pham et al., 2002), link position and/or velocity sensors (Tsaprounis and Aspragathos, 2000, Huang, 2003) or torque sensors (AlbuSchaffer and Hirzinger, 2001). Ostring et al., 2003 and Pham et al., 2001 have tried to avoid the use of additional sensors. Their approach is restricted to linear models, implying that nonlinear terms such as Coulomb friction and gravitation forces cannot be included in the dynamic model. Omitting these forces is not realistic, since joint friction accounts for a significant part of the motor torque of industrial robots. Therefore the application of additional sensors seems to be the most promising method to identify the dynamic parameters of flexible-joint robots. Within the scope of this paper it is assumed that all flexible deformations can be measured by for example a camera system as used by Yoshikawa et al., 2001.

In most of the literature about flexible-joint robots, only drive flexibilities are considered. Flexible joints are modelled as torsional springs (Spong, 1987). From static experiments on an industrial robot in our laboratory it appeared that the bending stiffness of the joints introduced by the bearings is of the same order as the drive stiffness. The problem of combined joint and drive flexibilities has not received much attention so far. However, for realistic dynamic simulations of robot tasks that demand high tracking accuracy combined with high operational speeds, the effects of both joint and drive flexibilities should be included in the model.

In this paper a nonlinear finite element method is used to generate dynamic equations of motion in terms of a mixed set of generalized coordinates describing joint angles and deformation parameters that characterize both joint and drive flexibilities. Joint friction is described by means of a static friction model, including Coulomb and viscous friction components. The equations of motion are written in a form linear in the dynamic parameters. This is to facilitate the use of linear least squares techniques for the identification of the parameters. Measurement data is collected from closed loop identification experiments in which the robot is programmed to follow a prescribed trajectory. To excite the flexibilities, feed forward torques with frequencies above the bandwidth of the feedback controller will be applied. The identification method will be demonstrated on simulated responses of a four-link flexible-joint manipulator. The results are validated by comparing computed tip motions of the original and the identified model. 


\section{Robot model}

In the finite element method presented, a manipulator is modelled as an assembly of finite elements; Jonker, 1990. This is illustrated in figure 1 where a 4 DOF manipulator is modelled using two different types of elements. The manipulator links are modelled as beam elements and the joints are modelled by hinge elements. The location of each element is described relative to a global inertial coordinate system by a set of nodal coordinates $x_{i}^{(k)}$. Some coordinates may be Cartesian coordinates of the end nodes, while others describe the orientation of orthogonal triads, rigidly attached to the element nodes. The superscript $k$ is added to show that a specific element $k$ is considered. With respect to some reference configuration of the element, the instantaneous values of the nodal coordinates represent a fixed number of deformation modes for the element. The deformation modes are specified by a set of deformation parameters $\boldsymbol{e}^{(k)}$, some of which are associated with large relative displacements and rotations between the element nodes, while others describe small elastic deformations of the element and will be denoted by $\boldsymbol{\epsilon}^{(k)}$. The number of deformation parameters is equal to the number of nodal coordinates minus the number of degrees of freedom of the element as a rigid body. Note that rigid body motions of the elements are characterized by displacements and rotations of the nodal points for which all deformations are zero. The components of the vector of deformation parameters $\left(\boldsymbol{e}^{(k)}, \boldsymbol{\epsilon}^{(k)}\right)$ can be expressed as analytic functions of the vector of nodal coordinates $\boldsymbol{x}^{(k)}$. In this way a vector function $\left(\boldsymbol{e}^{(k)}, \boldsymbol{\epsilon}^{(k)}\right)=\mathcal{D}^{(k)}\left(\boldsymbol{x}^{(k)}\right)$ can be defined for each element $k$.

\section{Link Model}

The manipulator links are modelled by beam elements. The configuration of the beam element is determined by the position vectors $\boldsymbol{x}^{p}$ and $\boldsymbol{x}^{q}$ of the end nodes and the angular orientation of orthogonal triads $\left(\boldsymbol{n}_{x^{\prime}}, \boldsymbol{n}_{y^{\prime}}, \boldsymbol{n}_{z^{\prime}}\right)$ rigidly attached to the element nodes as shown in figure 2 . The triads coin-

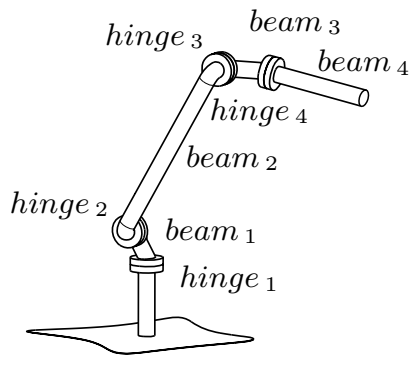

Figure 1. Model 4 DOF manipulator

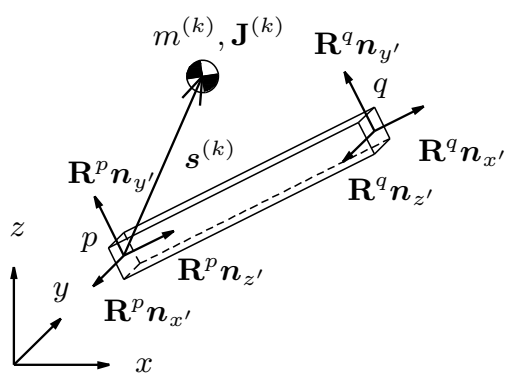

Figure 2. Link $k$ with lumped mass $m^{(k)}$ and rotational inertia $\mathbf{J}^{(k)}$ 
cide with the axis $p q$ and the principle axes of its cross section. The rotation part of the motion of the beam element is described by the rotation of the triads $\left(\boldsymbol{n}_{x^{\prime}}, \boldsymbol{n}_{y^{\prime}}, \boldsymbol{n}_{z^{\prime}}\right)$ which are determined by rotation matrices $\mathbf{R}^{p}$ and $\mathbf{R}^{q}$, respectively. Six deformation parameters are defined for the beam element. As the links are rigid, all deformations are prescribed to be zero. For a detailed description of the deformation functions $\mathcal{D}^{(k)}\left(\boldsymbol{x}^{(k)}\right)$, the reader is referred to Jonker, 1990.

The assumption of rigid links enables the use of a lumped mass description, in which the inertia and mass properties of a link are modelled by means of a lumped body attached at node $p$ of the beam element; see figure 2. The dynamic properties of each body are described by a symmetric rotational inertia matrix $\mathbf{J}^{(k)}$, a mass $m^{(k)}$ and a vector $\boldsymbol{s}^{(k)}$ defining the center of gravity with respect to the corresponding element node at which the body is lumped. For each link element, a lumped parameter vector $\boldsymbol{p}^{(l, k)}$ is defined as:

$$
\boldsymbol{p}^{(l, k)}=\left(m, m s_{x^{\prime}}, m s_{y^{\prime}}, m s_{z^{\prime}}, J_{x^{\prime} x^{\prime}}, J_{x^{\prime} y^{\prime}}, J_{x^{\prime} z^{\prime}}, J_{y^{\prime} y^{\prime}}, J_{y^{\prime} z^{\prime}}, J_{z^{\prime} z^{\prime}}\right)^{(k)}
$$

containing the components of $m^{(k)}, \boldsymbol{s}^{(k)}$ and $\mathbf{J}^{(k)}$ respectively. A detailed description can be found in Hardeman et al., 2005.

\section{Joint Model}

Each robot joint is modelled by two cylindrical hinge elements, a motor hinge element and a flexible hinge element, describing the joint and drive flexibilities; see figure 3. Each cylindrical hinge element is hereafter termed hinge element and has two nodes $p$ and $q$ at the element axis; see figure 4 . The configuration of the element is determined by two orthogonal triads $\left(\boldsymbol{n}_{x^{\prime}}, \boldsymbol{n}_{y^{\prime}}, \boldsymbol{n}_{z^{\prime}}\right)$ rigidly attached to each end node. For the hinge element, three deformation functions are defined, namely a large relative rotation about the element axis denoted $e_{1}^{(k)}$ and two small orthogonal bending deformations denoted $\epsilon_{2}^{(k)}$ and $\epsilon_{3}^{(k)}$ respectively. For a detailed description of the hinge element, the reader is referred to Geradin et al., 1986 and Schwab and Meijaard, 1999.

The motor hinge represents the driving system which generates the net driving torque $\tau^{(k)}$. The relative rotation $e_{1}^{(k)}$ is referred to as the joint angle. The bending deformations $\epsilon_{2}^{(k)}$ and $\epsilon_{3}^{(k)}$ of the motor hinge are prescribed to be zero. The net driving torque is the driving torque of the motor $\tau^{(m, k)}$ minus the torque needed to accelerate a motor inertia $J^{(m, k)}$ and a joint friction torque $\tau^{(f, k)}$ :

$$
\tau^{(k)}=\tau^{(m, k)}-J^{(m, k)} \ddot{e}_{1}^{(k)}-\tau^{(f, k)} .
$$

Joint friction is described using a static friction model containing Coulomb $c^{(c, k)}$ and viscous $c^{(v, k)}$ friction:

$$
\tau^{(f, k)}=c^{(c, k)} \operatorname{sign}\left(\dot{e}_{1}^{(k)}\right)+c^{(v, k)} \dot{e}_{1}^{(k)} .
$$




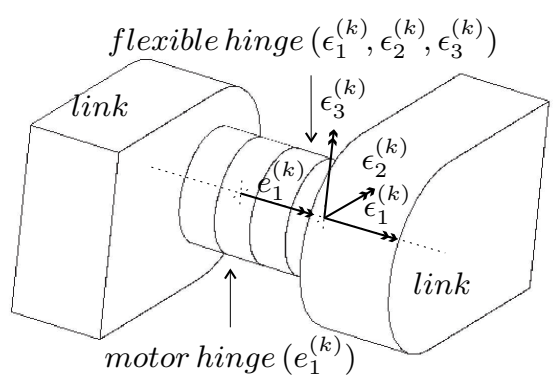

Figure 3. Joint model

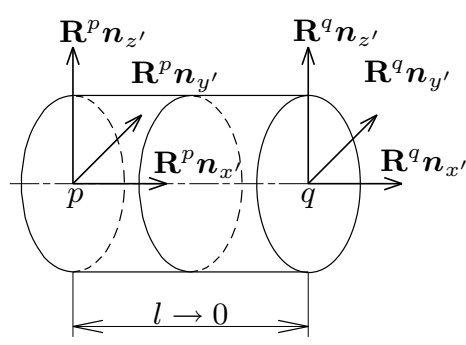

Figure 4. Hinge element

The joint and drive flexibilities are described by the flexible hinge element. The elastic deformations of the hinge are assumed to be small and are denoted by $\boldsymbol{\epsilon}^{(k)}=\left(\epsilon_{1}^{(k)}, \epsilon_{2}^{(k)}, \epsilon_{3}^{(k)}\right)$ for the drive flexibility and two orthogonal bending modes of the joint respectively. The vector of associated stress resultants $\boldsymbol{\sigma}^{(k)}$ is linearly related to the deformations $\boldsymbol{\epsilon}^{(k)}$ and their time derivatives $\dot{\boldsymbol{\epsilon}}^{(k)}$ by the constitutive equation:

$$
\boldsymbol{\sigma}^{(k)}=\mathbf{K}^{(k)} \boldsymbol{\epsilon}^{(k)}+\mathbf{D}^{(k)} \dot{\boldsymbol{\epsilon}}^{(k)},
$$

where $\mathbf{K}^{(k)}$ and $\mathbf{D}^{(k)}$ are diagonal matrices containing respectively the stiffness parameters $\boldsymbol{k}^{(k)}$ and structural damping parameters $\boldsymbol{d}^{(k)}$.

\section{Equations of motion}

A manipulator model is built up from finite elements by letting the elements have nodal points in common. The assembly of finite elements is realized by defining a vector $\boldsymbol{x}$ of nodal coordinates for the entire manipulator mechanism. The motion of the manipulator mechanism is described by relative degrees of freedom, which are the joint rotations, denoted by $\boldsymbol{e}^{(m)}$, as well as the elastic deformation parameters of the joints denoted by $\epsilon^{(m)}$. By means of the geometric transfer function $\mathcal{F}^{(x)}$, the nodal coordinates $\boldsymbol{x}$ are expressed as functions of the generalized coordinates $\boldsymbol{q}=\left(\boldsymbol{e}^{(m)}, \boldsymbol{\epsilon}^{(m)}\right)$, so

$$
\boldsymbol{x}=\mathcal{F}^{(x)}(\boldsymbol{q}) .
$$

Differentiating the transfer function with respect to time gives:

$$
\dot{\boldsymbol{x}}=\mathrm{D} \mathcal{F}^{(x)} \dot{\boldsymbol{q}},
$$

where the differentiation operator $\mathrm{D}$ represents partial differentiation with respect to the degrees of freedom. Differentiating again with respect to time yields the accelerations:

$$
\ddot{\boldsymbol{x}}=\mathrm{D} \mathcal{F}^{(x)} \ddot{\boldsymbol{q}}+\left(\mathrm{D}^{2} \mathcal{F}^{(x)} \dot{\boldsymbol{q}}\right) \dot{\boldsymbol{q}}
$$


The geometric transfer function $\mathcal{F}^{(x)}$ and the derivatives $\mathrm{D} \mathcal{F}^{(x)}$ and $\mathrm{D}^{2} \mathcal{F}^{(x)}$ are computed from the deformation functions of the finite elements and are determined numerically in an iterative way; see Jonker, 1990.

Let $\mathbf{M}$ be the global mass matrix and $\boldsymbol{f}$ be the vector of nodal forces including the velocity dependent inertia forces and the gravitational forces obtained by assembling the contributions of the lumped bodies of the links. Let $\boldsymbol{\tau}^{(m)}$ be the vector of driving torques, $\boldsymbol{\tau}^{(f)}$ be the global vector of friction torques and $\sigma$ be the global vector of stress resultants obtained by assembling the contributions of equation (2) and equation (4) respectively. Then the equations of motion described in terms of the degrees of freedom $\boldsymbol{q}$ are given by

$$
\left[\begin{array}{ll}
\overline{\mathbf{M}}^{e e} & \overline{\mathbf{M}}^{e \epsilon} \\
\overline{\mathbf{M}}^{\epsilon e} & \overline{\mathbf{M}}^{\epsilon \epsilon}
\end{array}\right]\left[\begin{array}{l}
\ddot{\boldsymbol{e}}^{(m)} \\
\ddot{\boldsymbol{\epsilon}}^{(m)}
\end{array}\right]+\left[\begin{array}{l}
\mathrm{D}_{e} \mathcal{F}^{(x) T} \\
\mathrm{D}_{\epsilon} \mathcal{F}^{(x) T}
\end{array}\right]\left[\mathbf{M}\left(\mathrm{D}^{2} \mathcal{F}^{(x)} \dot{\boldsymbol{q}}\right) \dot{\boldsymbol{q}}-\boldsymbol{f}\right]+\left[\begin{array}{c}
\boldsymbol{\tau}^{(f)} \\
\boldsymbol{\sigma}
\end{array}\right]=\left[\begin{array}{c}
\boldsymbol{\tau}^{(m)} \\
\mathbf{0}
\end{array}\right]
$$

where

$$
\begin{array}{ll}
\overline{\mathbf{M}}^{e e}=\mathrm{D}_{e e} \mathcal{F}^{(x) T} \mathbf{M D}_{e e} \mathcal{F}^{(x)}+\operatorname{diag}\left(\boldsymbol{J}^{(m)}\right), & \overline{\mathbf{M}}^{e \epsilon}=\mathrm{D}_{e \epsilon} \mathcal{F}^{(x) T} \mathbf{M D}_{e \epsilon} \mathcal{F}^{(x)} \\
\overline{\mathbf{M}}^{\epsilon e}=\mathrm{D}_{\epsilon e} \mathcal{F}^{(x) T} \mathbf{M D}_{\epsilon e} \mathcal{F}^{(x)}, & \overline{\mathbf{M}}^{\epsilon \epsilon}=\mathrm{D}_{\epsilon \epsilon} \mathcal{F}^{(x) T} \mathbf{M D}_{\epsilon \epsilon} \mathcal{F}^{(x)}
\end{array}
$$

are the reduced mass matrices. Note that matrix $\overline{\mathbf{M}}^{e e}$ also includes the vector of motor inertias $\boldsymbol{J}^{(m)}$. The above formulation of the equations of motion in acceleration linear form will be used for the forward dynamic simulations.

The equations of motion can also be written in a parameter linear form which will be used to estimate the dynamic parameters

$$
\boldsymbol{\Phi}(\boldsymbol{q}, \dot{\boldsymbol{q}}, \ddot{\boldsymbol{q}}) \boldsymbol{p}=\left[\begin{array}{c}
\boldsymbol{\tau}^{(m)} \\
\mathbf{0}
\end{array}\right]
$$

where

$$
\boldsymbol{\Phi}=\left[\begin{array}{ccccc}
\boldsymbol{\Phi}^{(l)} & \operatorname{diag}\left(\ddot{\boldsymbol{e}}^{(m)}\right) \operatorname{diag}\left(\dot{\boldsymbol{e}}^{(m)}\right) \operatorname{diag}\left(\operatorname{sign}\left(\dot{\boldsymbol{e}}^{(m)}\right)\right) & \mathbf{0} & \mathbf{0} \\
\mathbf{0} & \mathbf{0} & \mathbf{0} & \operatorname{diag}\left(\boldsymbol{\epsilon}^{(m)}\right) & \operatorname{diag}\left(\dot{\boldsymbol{\epsilon}}^{(m)}\right)
\end{array}\right]
$$

and parameter vector $\boldsymbol{p}$ is defined as

$$
\boldsymbol{p}=\left(\boldsymbol{p}^{(l)}, \boldsymbol{J}^{(m)}, \boldsymbol{c}^{(c)}, \boldsymbol{c}^{(v)}, \boldsymbol{k}, \boldsymbol{d}\right) .
$$

Vector $\boldsymbol{p}^{(l)}$ includes the lumped mass and inertia parameters of the robot links. Furthermore $\boldsymbol{J}^{(m)}, \boldsymbol{c}^{(c)}, \boldsymbol{c}^{(v)}, \boldsymbol{k}, \boldsymbol{d}$ represent the parameter vectors containing motor inertias, Coulomb friction, viscous friction, stiffness and structural damping coefficients, respectively. For a detailed description of the matrix $\Phi^{(l)}$ the reader is referred to Hardeman et al., 2005. 


\section{Identification method}

The linear least squares technique will be used to identify the unknown dynamic parameters of a robot. During an identification experiment, the robot is moved along a prescribed trajectory while the joint driving torques $\boldsymbol{\tau}^{(m)}$ and the degrees of freedom $\boldsymbol{q}$ are measured. The velocities $\dot{\boldsymbol{q}}$ and accelerations $\ddot{\boldsymbol{q}}$ will be obtained afterwards by numerical differentiation. The driving torques $\boldsymbol{\tau}^{(m)}\left(t_{i}\right)$ and the system matrices $\boldsymbol{\Phi}\left(t_{i}\right)$ at all time steps $t_{i}=1 \cdots N$ are stacked in vector $\boldsymbol{y}$ and regression matrix $\mathbf{A}$ respectively:

$$
\boldsymbol{y}=\left[\begin{array}{c}
\boldsymbol{\tau}\left(t_{1}\right) \\
\mathbf{0} \\
\vdots \\
\boldsymbol{\tau}\left(t_{N}\right) \\
\mathbf{0}
\end{array}\right], \quad \mathbf{A}=\left[\begin{array}{c}
\boldsymbol{\Phi}\left(t_{1}\right) \\
\vdots \\
\boldsymbol{\Phi}\left(t_{N}\right)
\end{array}\right]
$$

yielding the equation:

$$
\boldsymbol{y}=\mathbf{A} p+\rho,
$$

where the vector $\rho$ represents the residual torques arising from measurement noise and model errors. Given the measurements $\boldsymbol{y}$ and the regression matrix A the dynamic parameters are identified by solving the linear least squares problem

$$
\hat{\boldsymbol{p}}=\arg \min _{\boldsymbol{p}}\|\boldsymbol{y}-\mathbf{A} \boldsymbol{p}\|^{2} .
$$

The solution can be found by using the concept of the pseudo-inverse

$$
\hat{\boldsymbol{p}}=\mathbf{A}^{+} \boldsymbol{y}
$$

where $\mathbf{A}^{+}$represents the pseudo-inverse of $\mathbf{A}$ that can be calculated from a Singular Value Decomposition of the regression matrix A; see Lawson and Hanson, 1974.

Not all parameters of the vector $\boldsymbol{p}$ can be identified, since some of the parameters do not affect the dynamic response at all or affect the dynamic response only in linear combinations with other parameters. Application of the Singular Value Decomposition gives insight into the maximum number of identifiable parameters and parameters that show up in linear combinations. Both depend on the actual model, the size of the parameters, the excitation signal and the amount of measurement noise.

The design of an identification experiment includes the choice of input and output signals. With the experimental limitations in mind, the following approach is suggested. Parameters related to position dependent behavior such as gravitation can only be identified when measurement data is obtained from sufficiently distinguishable robot configurations. Therefore, the robot is programmed to follow a trajectory $\boldsymbol{r}$ along various configurations in its work envelope. For stability and safety reasons, the experiments will be carried out in 


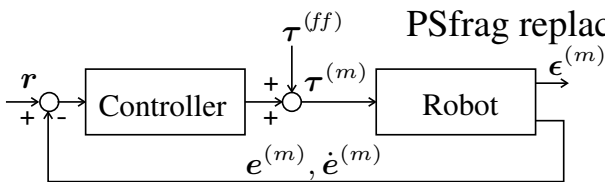

Figure 5. Closed loop system

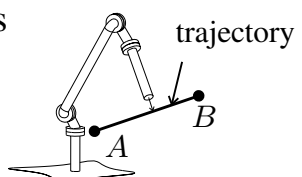

Figure 6. Validation trajectory

closed loop. Problems arise for the identifiability of the dynamic parameters of the flexible joints because the controller is designed to damp out the flexibilities of the robot joints. Therefore, during the trajectory the driving torques are perturbed with feed forward torques $\tau^{(f f)}$ having a frequency spectrum beyond the bandwidth of the closed-loop system; see figure 5 .

\section{Results and discussion}

The identification method will be demonstrated on the basis of simulated responses of the four-link manipulator presented in figure 1 . The dynamic parameters of the robot model are adopted from a real industrial robot. The mass and inertia parameters are obtained from CAD data. Friction parameters are found by measuring the joint torque during constant velocity motion. The stiffness parameters are estimated by measuring the deformations of the joints as functions of static loads applied on the robot arms. A relative damping equal to $1 \%$ is assumed for each vibrational mode. The dynamic responses are computed in a closed-loop simulation with a model of a real digital PID controller; see figure 5. The bandwidth of the closed loop system is about 15 Hz.

The robot performs an identification trajectory where each joint angle varies between 0 and $\frac{1}{2} \pi$ according to a sine function with a period of $4.1 \mathrm{~s}$. The joint flexibilities are excited by means of a feed forward torque described by a multi sine signal containing 204 frequencies ranging from $0.12 \mathrm{~Hz}$ to $50 \mathrm{~Hz}$. A multi sine signal has been used in order to obtain a sharp cutoff for frequencies above $50 \mathrm{~Hz}$. This is required because in the actual controller, the torque and position data are sampled at $250 \mathrm{~Hz}$ without any aliasing filter. The velocities and accelerations are obtained by means of numerical differentiation of the position signals using an eight order central difference scheme given by Khan and Ohba, 2003.

From the simulated responses, the regression matrix $\mathbf{A}$ and the measurement vector $\boldsymbol{y}$ are constructed. The parameter vector $\boldsymbol{p}$ contains 76 parameters, namely 10 lumped inertia parameters $\boldsymbol{p}^{(l, k)}$ for each link and 1 motor inertia, 2 friction parameters, 3 stiffness and 3 damping parameters for each joint. Using a Singular Value Decomposition of the regression matrix, a parameter set with 64 linearly independent parameters has been identified from the data. 


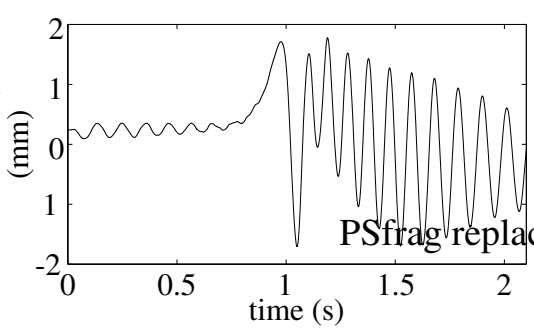

Figure 7a. Vertical deviation robot tip original model

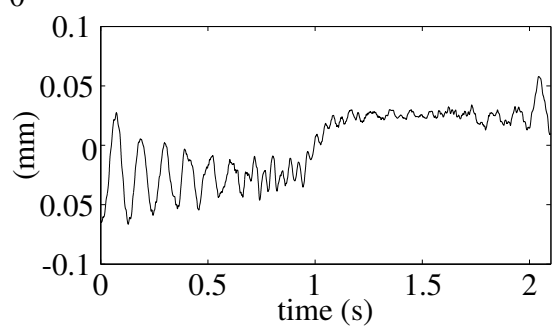

Figure $7 c$. Difference between tip motion of original and identified model in vertical direction

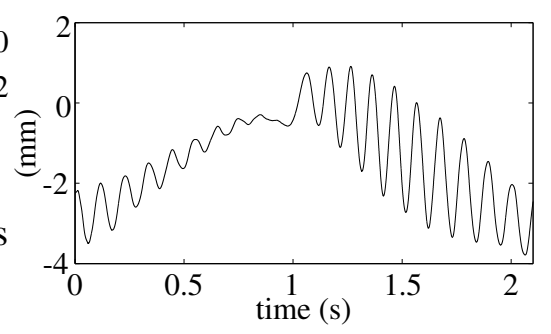

Figure $7 b$. Horizontal deviation robot tip original model

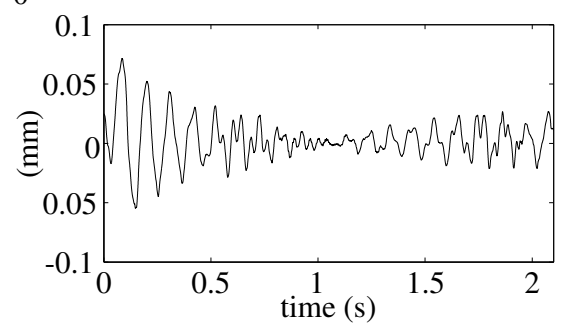

Figure $7 d$. Difference between tip motion of original and identified model in horizontal direction

Simulated tip responses of both the original and the identified model are used for validation of the identified parameters. The robot is programmed to perform a tip motion along a straight line of $1.7 \mathrm{~m}$ in the horizontal plane as illustrated in figure 6 with starting point $A$ and endpoint $B$. Due to the flexibilities and tracking errors of the control system, the robot tip will deviate from the straight line. The tip deviations of the original model are shown in figures $7 \mathrm{a}$ and $7 \mathrm{~b}$ in vertical direction and horizontal directions, respectively. Figures $7 \mathrm{c}$ and $7 \mathrm{~d}$ show the differences between the tip motion of the original and the identified model again in vertical and horizontal direction. These differences appear to be at least an order of magnitude smaller than the amplitudes of the tip deviations of the original model. Similar results are found when other quantities are compared, e.g. the driving torques. It can be concluded that there is a good agreement between both models.

\section{Conclusion}

In this paper, we have provided a dynamic robot model describing both drive and joint flexibilities. A nonlinear finite element based method is used to derive the dynamic equations of motion in a form suitable for both simulation and identification. The presented numerical example of the four-link manipulator 
showed that the proposed identification method gives useful results, at least in simulations. In future research, the identification will be carried out with experimental data from a real industrial robot.

\section{Acknowledgments}

This research was carried out under project number MC8.03161 in the framework of the Strategic Research programme of the Netherlands Institute for Metals Research in the Netherlands (www.nimr.nl).

\section{References}

Albu-Schaffer, A. and Hirzinger, G. (2001). Parameter identification and passivity based joint control for a 7 dof torque controlled light weight robot. In IEEE International Conference on Robotics and Automation, pages 2852-2858.

Geradin, M., Robert, G., and Buchet, P. (1986). Finite Element Methods for Nonlinear Problems, chapter Kinematic and Dynamic Analysis of Mechanisms a Fininite Element Approach Based on Euler Parameters. Springer-Verlag, Berlin.

Hardeman, T., Aarts, R.G.K.M., and Jonker, J.B. (2005). A finite element formulation for dynamic parameter identification of robot manipulators. Submitted to Multibody System Dynamics.

Huang, J.T. (2003). A new approach to parametric identification of a single-link flexible joint manipulator. Jounal of Intelligent and Robotic Systems, 37:273-284.

Jonker, J.B. (1990). A finite element dynamic analysis of flexible manipulators. International Journal of Robotic Research, 9:59-74.

Khan, I.R. and Ohba, R. (2003). Taylor series based finite differentce approximations of higherdegree derivatives. Journal of Computational and Applied Mathematics, 154:115-124.

Lawson, C.L. and Hanson, R.J. (1974). Solving Least Squares Problems. Prentice-Hall. Englewood Cliffs, New Jersey.

Ostring, M, Gunnarsson, S., and Norrlef, M. (2003). Closed-loop identification of an industrial robot containing flexibilities. Control Engineering Practice, 11:291-300.

Pham, M.T., Gautier, M., and Poignet, Ph. (2001). Identification of joint stiffness with bandpass filtering. In IEEE International Conference on Robotics and Automation, pages 2867-2872.

Pham, M.T., Gautier, M., and Poignet, Ph. (2002). Accelerometer based identification of mechanical systems. In IEEE International Conference on Robotics and Automation, pages 4293-4298.

Schwab, A.L. and Meijaard, J.P. (1999). The belt, gear, bearing and hinge as special finite elements for kinematic and dynamic analysis of mechanisms and machines. In Dynamics, Nonlinear Oscillations, Rotor Dynamics and Software development.

Spong, M.W. (1987). Modeling and control of elastic joint robots. Journal of Dynamic Systems, Measurement, and Control, 109:310-319.

Tsaprounis, C.J. and Aspragathos, N.A. (2000). Adaptive tracking controller for rigid-link elasticjoint robots with link acceleration astimation. Journal of Intelligent and Robotic Systems, 27:68-83.

Yoshikawa, T., Ohta, A., and Kanaoko, K. (2001). State estimation and parameter identification of flexible manipulators based on visual sensor and virtual joint method. In IEEE International Conference on Robotics and Automation, pages 2840-2845. 